\title{
Cribriform adenocarcinoma of the base of the tongue and low-grade, polymorphic adenocarcinomas of the salivary glands
}

\author{
ALEŠ ČOČEK ${ }^{1}$, KLÁRA HRONKOVÁ ${ }^{1}$, JANA VOLDÁNOVÁ ${ }^{1}$, JOSEF ŠACH ${ }^{2}$, \\ ALENA SKÁLOVÁ ${ }^{5}$, MILOSLAV AMBRUŠ ${ }^{3}$, JANA VRÁNOVÁ ${ }^{4}$ and ALEŠ HAHN ${ }^{1}$ \\ Departments of ${ }^{1}$ Otorhinolaryngology, ${ }^{2}$ Pathology, ${ }^{3}$ Radiotherapy and Oncology and \\ ${ }^{4}$ Medical Biophysics and Medical Informatics, Third Faculty of Medicine, Charles University in Prague, \\ Faculty Hospital Královské Vinohrady, Prague; ${ }^{5}$ Laboratory of Biopsy, Ltd., Pilsen, Czech Republic
}

Received July 29, 2010; Accepted October 22, 2010

DOI: $10.3892 / \mathrm{ol} .2010 .213$

\begin{abstract}
Low-grade, polymorphic adenocarcinomas occur mainly in females and are usually associated with the small salivary glands of the palate. The tumors are malignant, but not aggressive. Regional neck as well as distant metastasis is rare and the mortality rate is low. Cribriform adenocarcinoma of the salivary glands is a rare tumor, currently ranked among low-grade, polymorphic adenocarcinomas of the salivary glands. However, it differs from carcinomas in this group as it metastasizes to the cervical lymph nodes and exhibits frequent primary localization in the small salivary glands at the base of the tongue. Despite the tendency to metastasize, patient prognosis remains favorable. A case of a 72-year-old woman with neck metastases of cribriform adenocarcinoma, of unknown primary origin, is reported. The primary tumor origin was ultimately determined using nuclear magnetic resonance, histological verification was difficult due to the presence of an intact mucosal cover over the tumor. Cribriform adenocarcinoma is known to have a number of characteristics in common with a typical low-grade, salivary gland adenocarcinoma. However, in contrast to low-grade adenocarcinomas, the tumor presented with neck lymph node metastasis.
\end{abstract}

\section{Introduction}

Cribriform adenocarcinoma of the small salivary glands is defined as a low-grade, polymorphic adenocarcinoma based on the World Health Organization histopathological classification of carcinomas of the salivary glands (2005, Table I). The expression 'low-grade, polymorphic adenocarcinoma',

Correspondence to: Dr Aleš Čoček, Department of Otorhinolaryngology, Third Faculty of Medicine, Charles University in Prague, Faculty Hospital Královské Vinohrady, 10034, Prague 10, Czech Republic

E-mail: ales.cocek@seznam.cz

Key words: salivary glands tumors, base of tongue, cribriform adenocarcinoma, epithelial markers, nuclear magnetic resonance originally termed 'terminal duct carcinoma', was introduced by Batsakis and Evans in 1983 (1,2). It occurs primarily in females $(70 \%)$, usually older than 60 years of age, and more often in Africans and African-Americans. It is a tumor that usually arises from the small salivary glands of the palate and is microscopically unconfined (3). Tumor architecture is varied in that cells have a regular round, elongated or ovoid nucleus with a benign appearance. The nucleus is frequently vacualized, causing it to resemble a papillary carcinoma of the thyroid gland, particularly in tumors associated with the small salivary glands at the base of tongue (4). Tumors generally exhibit a low mitotic activity without an atypical appearance. Immunohistochemically, the tumors are positive for epithelial tumor markers, particularly cytokeratin and epithelial membrane antigen, S-100 and $\alpha$-SMA. The MIB 1 proliferative index is low. The oncoprotein BCL-2 is expressed, but the expression of tumor protein p53 is lacking (5). The tumors are malignant, but not aggressive. Regional neck as well as distant metastasis is rare and the mortality rate is low. For these reasons, the accepted therapeutic method is radical surgical removal without elective neck dissection or post-operative actinotherapy (6).

Cribriform adenocarcinoma of the small salivary glands at the base of the tongue, as well as low-grade polymorphic cancers, are unbounded tumors that are strongly S100-positive. These tumors exhibit negative staining for the thyroid gland markers TTF1 and TG. However, they differ from low-grade polymorphic adenocarcinomas in that they metastasize to the lymph nodes of the neck. While metastasis is already present at the time of diagnosis, the prognosis remains positive.

\section{Case Report}

Patient. A 72-year-old female was examined at the ENT clinic for a level II, right-side neck mass that had been present for 1 year, but without recent progression. Neck ultrasonography showed a cystic formation of $4 \times 3 \times 1.5 \mathrm{~cm}$. The patient underwent thyroid gland surgery in 1991, in another clinic, and sonography showed residue in the bed of the right lobe of the gland. The patient had also undergone a childhood tonsillectomy for recurrent tonsillitis. The cystic mass was extirpated; macroscopically it appeared cystic with dark contents. 
Table I. World Health Organisation histopathological classification of carcinomas of the salivary glands (2005).

\begin{tabular}{ll}
\hline Acinic cell carcinoma & $8550 / 3$ \\
Mucoepidermoid carcinoma & $8430 / 3$ \\
Adenoid cystic carcinoma & $8200 / 3$ \\
Polymorphous low-grade adenocarcinoma & $8525 / 3$ \\
Epithelial-myoepithelial carcinoma & $8562 / 3$ \\
Clear cell carcinoma, not otherwise specified & $8310 / 3$ \\
Basal cell adenocarcinoma & $8147 / 3$ \\
Sebaceous carcinoma & $8410 / 3$ \\
Sebaceous lymphadenocarcinoma & $8410 / 3$ \\
Cystadenocarcinoma & $8440 / 3$ \\
Low-grade cribriform cystadenocarcinoma & \\
Mucinous adenocarcinoma & $8480 / 3$ \\
Oncocytic carcinoma & $8290 / 3$ \\
Salivary duct carcinoma & $8500 / 3$ \\
Adenocarcinoma, not otherwise specified & $8140 / 3$ \\
Myoepithelial carcinoma & $8982 / 3$ \\
Carcinoma ex pleomorphic adenoma & $8941 / 3$ \\
Carcinosarcoma & $8980 / 3$ \\
Metastasizing pleomorphic adenoma & $8940 / 1$ \\
Squamous cell carcinoma & $8070 / 3$ \\
Small cell carcinoma & $8041 / 3$ \\
Large cell carcinoma & $8012 / 3$ \\
Lymphoepithelial carcinoma & $8082 / 3$ \\
Sialoblastoma & $8974 / 1$ \\
\hline
\end{tabular}

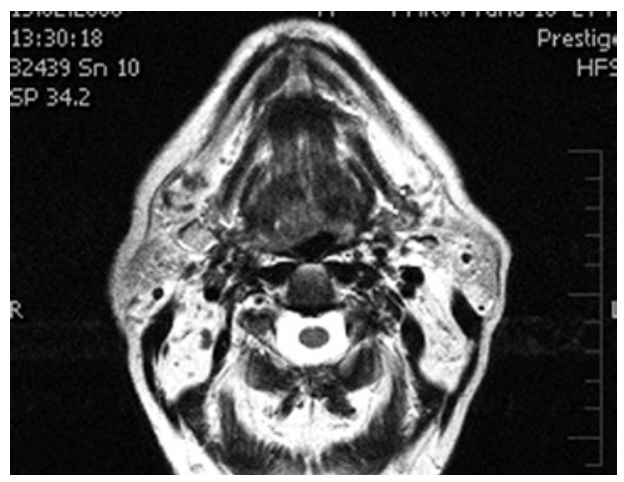

Figure 1. Nuclear magnetic resonance scan of the base of the tongue. Poorlydifferentiable infiltration is visible at the base of the right half of the tongue.

Methods. Initial histology suggested a well-differentiated metastasis of a myoepithelial cancer of the salivary glands. Tissue was sent for further histological examination to a laboratory specializing in the histological examination of salivary glands. Nuclear magnetic resonance was carried out, where an unconfined, poorly differentiable infiltration was found at the base of the right half of the tongue (Fig. 1). An endoscopic examination was performed and multiple biopsies were removed from the right side of the tongue base; however, the biopsies were histologically negative. A second endoscopic

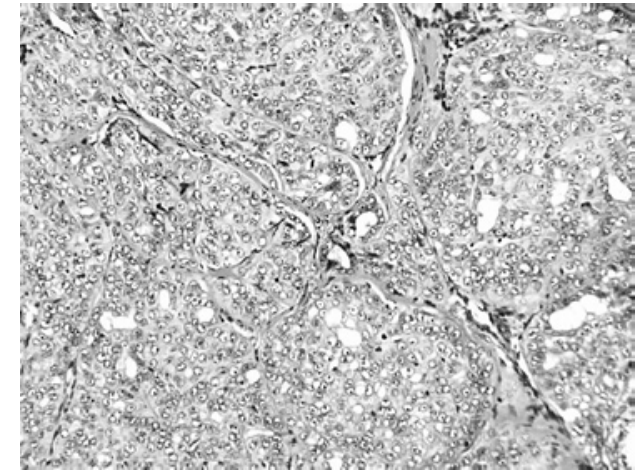

Figure 2. Cribriform arrangement of the tumor. Paraffin section, H\&E stain, magnification, $\mathrm{x} 125$.

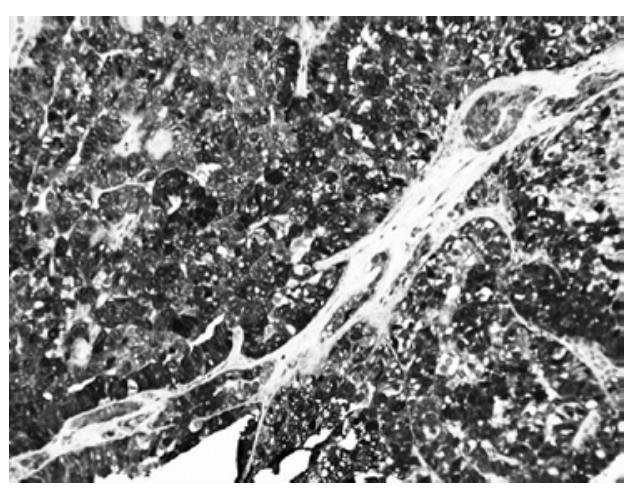

Figure 3. High positivity for the S100 protein. Paraffin section, magnification, $\mathrm{x} 125$.

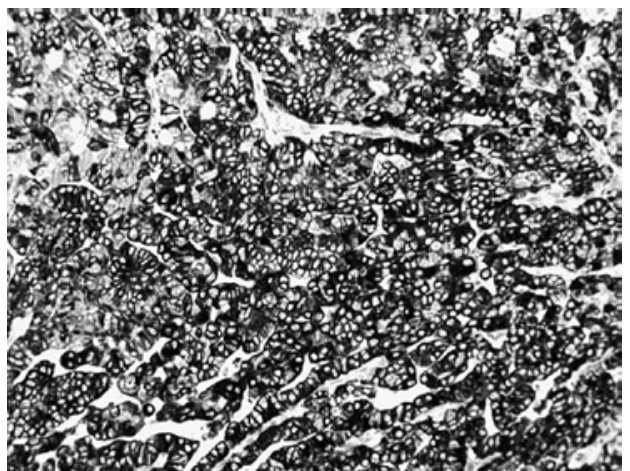

Figure 4. High positivity for the epithelial marker cytokeratin CK7. Paraffin section, magnification, $\mathrm{x} 125$.

biopsy was performed, and again the histological findings were negative. Given the circumstances, a right side neck dissection of cervical lymph nodes, sparing cranial nerve XI, and a lateral pharyngotomy with repeated preoperative biopsies, from the base of the tongue, was performed. The results revealed an adenocarcinoma at the base of the tongue. A resection of the tumor at the base of the tongue was performed together with a temporary tracheostomy. The tracheostomy was discontinued post-operatively and the patient was scheduled for post-operative radiotherapy due to the neck metastasis. Further histological investigation of the peroperative biopsies together with the neck metastases confirmed the diagnosis of 


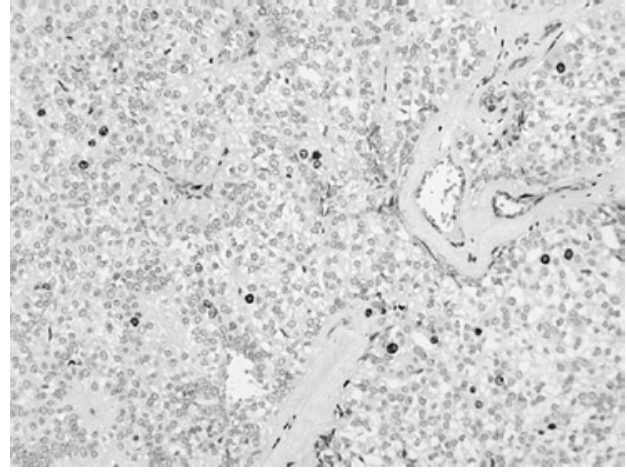

Figure 5. Proliferative marker MIB 1 (Ki-67). Paraffin section, magnification, $\mathrm{x} 125$.

rare cribriform adenocarcinoma of the small salivary glands at the base of the tongue. The patient is currently 3 years postradiotherapy without any local or regional recurrence of the disease.

\section{Results}

Histological findings. Findings from the histological examinations are shown in Figs. 1-8. The cribriform arrangement of the tumor is shown in Fig. 2. The tumor was highly positive for the S100 protein (Fig. 3) and the epithelial marker cytokeratin CK 7 (Fig. 4). Proliferative activity of the tumor was low [proliferative marker MIB 1 (Ki-67)] (Fig. 5). The tumor stained negative for actin (Fig. 6) and thyroid tumor markers TTF1 and TG were also negative. Fig. 7 shows the histology of the neck lymph node metastases. Fig. 8 shows the intact epithelial surface of the base of the tongue where the unconfined tumor developed, rendering the imaging examinations a challenge.

\section{Discussion}

Cribriform adenocarcinoma of small salivary glands is a rare tumor. According to the current WHO classification (2005), these tumors are included among low-grade polymorphic carcinomas, despite a strong tendency to metastasize to the cervical nodes, as shown in our case study and noted in the literature (4). While low-grade polymorphic carcinomas usually arise from small salivary glands of the palate, cribriform adenocarcinomas often arise from the small salivary glands at the base of tongue. This is highly significant in cases where the patient presents with neck metastasis from a tumor of unknown origin. If a diagnosis of metastasis from a cribriform adenocarcinoma is established by histopathological examination, it is reasonable and prudent to assume that the primary tumor is located at the base of the tongue and focus attention on this region. However, as noted in our case report, obtaining a positive histological result from the base of the tongue is challenging since the tumor grows unconfined under an intact epithelial surface (Fig. 8). Insufficiently deep excisions explain the negative histology. Additionally, the unconfined infiltration of the tumor renders imaging examinations challenging.

In conclusion, cribriform adenocarcinoma is currently included in a group with low-grade, polymorphic adenocarci-

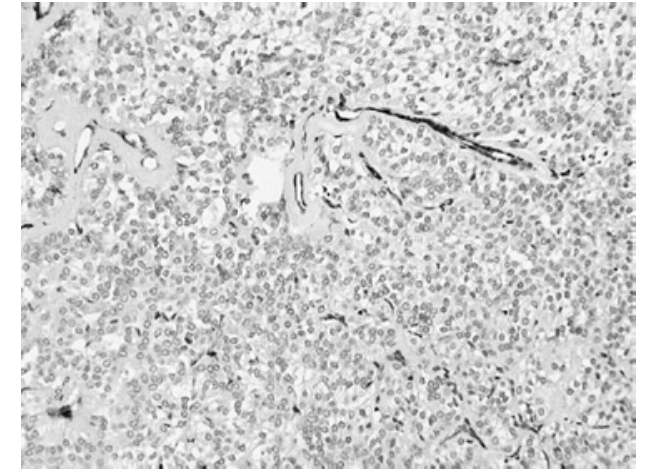

Figure 6. Negativity for actin. Paraffin section, magnification, x125.

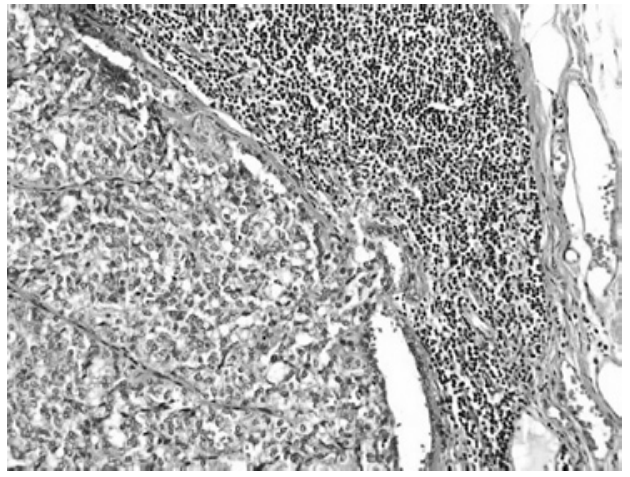

Figure 7. Histology of the neck lymph node metastases. Paraffin section, $\mathrm{H} \& \mathrm{E}$ stain, magnification, $\mathrm{x} 125$.

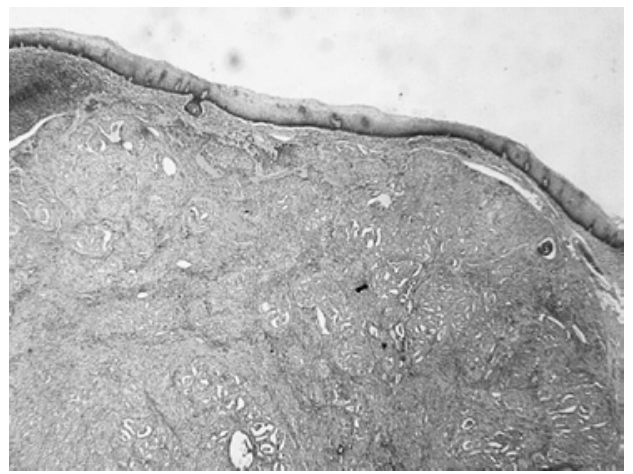

Figure 8. Intact epithelial surface of the base of tongue. Paraffin section, $\mathrm{H} \& \mathrm{E}$ stain, magnification, $\mathrm{x} 12.5$

nomas of the salivary glands. Cribriform adenocarcinoma has numerous characteristics in common with typical low-grade, polymorphic adenocarcinomas, including unlimited growth, low mitotic activity (low proliferative index of MIB 1), positive epithelial markers (particularly S-100 and cytokeratin), a resemblance to papillary carcinoma of the thyroid gland (although TTF1 and TG markers are negative), expression of the BCL-2 oncoprotein, without expression of tumor protein p53 and an equally favorable prognosis. Cribriform adenocarcinomas differ, however, as they metastasize to the cervical lymph nodes and exhibit frequent primary localization in the small salivary glands at the base of the tongue. This tendency is in contrast to low-grade, polymorphic adenocarcinomas that 
are associated with the small salivary glands of the palate and a negligible tendency towards neck lymph node metastasis. In light of neck metastasis, neck dissection and post-operative radiotherapy for cribriform adenocarcinoma are imperative. The magnitude and implications of the differences between the above-mentioned conditions may result in the removal of this tumor from the group of low-grade polymorphic adenocarcinomas and its reclassification as a separate entity with distinctive biological attributes.

\section{References}

1. Batsakis JG, Pinkston GR, Luna M, et al: Adenocarcinomas of the oral cavity: a clinicopathologic study of terminal duct carcinomas. J Laryngol Otol 97: 825-835, 1983.
2. Evans HL and Batsakis JG: Polymorphous low-grade adenocarcinoma of minor salivary glands: a study of 14 cases of a distinctive neoplasm. Cancer 53: 935-942, 1984.

3. Ellis GL and Auclair PL: Tumors of the salivary glands. In: Atlas of Tumor Pathology. Ellis GL (ed). AFIP, Washington DC, pp216-228, 1996.

4. Michal M, Skálová A, Simpson RH, et al: Cribriform adenocarcinoma of the tongue: a hitherto unrecognized type of adenocarcinoma characteristically occurring in the tongue. Histopathology 35: 495-501, 1999.

5. Perez-Ordonez B, Linkov I and Huvos AG: Polymorphous low-grade adenocarcinome of minor salivary glands: a study of 17 causes with emphasis on cell differentiation. Histopathology 32: 521-529, 1998

6. Wenig BM and Gnepp DR: Polymorphous low-grade adenocarcinoma of minor salivary glands. In: Surgical Pathology of the Salivary Glands. Ellis GL, Auclair PL and Gnepp DR (eds). Saunderes, Philadelphia, pp390-411, 1999. 